\title{
Long term neurological outcome of herpes encephalitis
}

\author{
E Lahat, J Barr, G Barkai, G Paret, N Brand, A Barzilai
}

\begin{abstract}
Twenty eight children with herpes simplex encephalitis were followed up for a mean of 5.5 years. Two children died and 26 survived, of whom 16 were left with no neurological sequelae and 10 had persistent neurological sequelae. Mean (SD) Glasgow coma score was significantly lower in the patients with neurological sequelae (7.7 (1.5)) and the patients who died (4.5 (0.7)), compared with the patients without neurological sequelae (11 (1.7)). (Arch Dis Child 1999;80:69-71)
\end{abstract}

Keywords: herpes simplex; encephalitis

Herpes simplex encephalitis (HSE) is an uncommon but severe disease with high mortality and morbidity. ${ }^{1}$ Estimates of the incidence range from two to four cases per million population each year. ${ }^{2}$ Approximately $30 \%$ of these occur in children, either in the neonatal period, acquired from maternal genital tract infection, or as a sporadic encephalitis in older children. ${ }^{2}$ The diagnosis of the neonatal form can sometimes be made by isolating the herpes simplex virus (HSV) from the cerebrospinal fluid (CSF), however in older children or adults, viral cultures of CSF are typically sterile. ${ }^{2}$ Many studies have documented the specific production of anti-HSV antibodies in CSF of patients with HSE. ${ }^{3}$ These tests have been shown to be specifically useful in children, however the antibody rise is generally detected later during the illness. ${ }^{3}$ For many years in these patients clinically suspected to have HSE, brain biopsy, despite many controversies, ${ }^{45}$ was the only way to obtain early and accurate confirmation of the diagnosis. ${ }^{2}$ In recent years, the use of polymerase chain reaction (PCR) to detect HSV DNA in $\mathrm{CSF}^{1}$ was introduced as an accurate method for early confirmation of the diagnosis of HSE. An effective and safe treatment, aciclovir, has been available since 1983, and its early use can significantly improve outcome, ${ }^{6}$ however considerable diagnostic difficulties, particularly in children, may lead to delay in treatment.

The purpose of our study was to investigate all children with HSE who were treated with aciclovir focusing on the clinical course of early and long term neurological sequelae.

\section{Patients and methods}

STUDY POPULATION

Patients aged between 9 months and 16 years with a diagnosis of HSE, who were treated in the paediatric department of the two university affiliated medical centres near Tel Aviv, Israel
(Assaf Harofeh and Sheba, Tel Hashomer) from January 1984 until June 1995 were included in the study.

\section{STUDY DESIGN}

Inpatient and outpatient charts of all patients with HSE were examined. Included in the study group were all patients treated with aciclovir, $30 \mathrm{mg} / \mathrm{kg} / \mathrm{day}$, divided into three daily doses given intravenously at eight hour intervals for 10 days.

The treatment was started after admission to the ward once a lumbar puncture had been done, and continued in all cases with a verified diagnosis of HSE.

All the patients who survived underwent a neurological evaluation before discharge. $\mathrm{Pa}-$ tients who were followed up in the paediatric neurology clinic for more than two years were included in the study group. All patients were examined in the clinic at least once every 4-6 months.

\section{CLINICAL OBSERVATIONS}

Data about the clinical assessment on admission, including pertinent history and full physical and neurological examination were obtained, focusing on duration, progression, and severity of central nervous system disease. The level of consciousness was evaluated according to the Glasgow coma scale.

Neurological evaluation was done before discharge and subsequently on each visit to the clinic (every $4-6$ months) by the same paediatric neurologist (EL).

Intellectual ability was assessed using the Wechsler intelligence scale for childrenrevised (WISC-R), and verbal, performance, and fullscale IQ were calculated.

LABORATORY DATA AND HSE VERIFICATION Initial CSF and serum samples were obtained from each patient at the beginning of treatment. Repeat CSF examinations were performed 5-7 days later. All samples were sent for routine studies including complete blood count, liver and kidney function tests, concentration of protein, glucose, and cell count of CSF.

Verification of HSE was based on fourfold increase in anti-HSV antibody CSF titres between the two samples, or positive CSF HSV $\operatorname{IgM}$ and IgG by enzyme-linked immunosorbent assay (ELISA). These measures of identification were taken from 1984 until April 1992 when the technique of polymerase chain reaction (PCR) to detect $\mathrm{HSV}$ DNA in CSF was introduced.

Data were collected in dBASE IV, a database computer program. Statistical analysis 
Table 1 Demographic and clinical data of children with HSE $(n=28)$

\begin{tabular}{lr}
\hline & No (\%) \\
\hline Sex & \\
Male & $22(79)$ \\
Female & $6(21)$ \\
Age & \\
$3-12$ months & $6(21)$ \\
$1-6$ years & $5(18)$ \\
$6-12$ years & $12(43)$ \\
$12-16$ years & $5(18)$ \\
Duration of symptoms before admission & \\
$<1$ day & $12(43)$ \\
$1-2$ days & $8(29)$ \\
$>2$ days & $8(29)$ \\
Symptoms on and before admission & \\
Fever $\left(>38^{\circ} \mathrm{C}\right)$ & $22(79)$ \\
Altered consciousness & $19(68)$ \\
Personality changes & $12(43)$ \\
Headache & $14(50)$ \\
Vomiting & $16(57)$ \\
Seizures & $19(68)$ \\
Generalised & $12(63)$ \\
Focal & $7(37)$ \\
Mean (SD) Glasgow coma score & $9.25(2.75)$ \\
\end{tabular}

(descriptive statistics and two sample $t$ test) was done with Statistix version 3.1 (Analytical Software, St Paul, Minnesota, USA) ( $p<0.05$ was considered significant).

\section{Results}

A total of 28 children were included in the study group. There were 22 boys and six girls, aged between 9 months and 16 years, with a mean (SD) age of 7.16 (4.84) years.

Table 1 summarises the demographic and clinical data on admission. Only six (21\%) of the patients were younger than 1 year old and the majority of them $(61 \%)$ were older than 6 years.

Duration of symptoms in almost half of the patients was less than one day, and the most common symptoms were fever $(n=22,79 \%)$, altered consciousness $(\mathrm{n}=19,68 \%)$, and seizures $(\mathrm{n}=19,68 \%)$. Only two patients had a history of labial herpes. Electroencephalogram (EEG) studies obtained for all patients during the first 24 hours after admission were abnormal in $24(86 \%)$ patients, demonstrating generalised slowing (10 patients), focal slowing (seven patients), and unilateral paroxysmal lateral epileptiform discharges (seven patients), whereas neuroimaging studies done at the same time were abnormal in only $10(36 \%)$ patients.

Abnormal white blood cell count in the CSF was found in $22(79 \%)$ of the patients, protein was increased in $20(71 \%)$, and glucose was slightly deceased in two $(7 \%)$.

Table 2 Clinical characteristics on admission of children with HSE in correlation to their long term neurological sequelae

\begin{tabular}{llll}
\hline & $\begin{array}{l}\text { No neurological } \\
\text { sequelae }(n=16)\end{array}$ & $\begin{array}{l}\text { Neurological sequelae } \\
(n=10)\end{array}$ & Died $(n=2)$ \\
\hline Mean (SD) age (years) & $8.66(5.07)$ & $4.6(3.9)$ & $8.0(1.4)$ \\
Duration of symptoms & 7 & 8 & 2 \\
$\quad<1$ day & 5 & - & - \\
$\quad$ 1-2 days & 4 & 2 & - \\
$\quad$ 2 days & 9 & 7 & 2 \\
Seizures & $11.06(1.69)$ & $7.7(1.49)$ & $4.5(0.7)$ \\
Mean (SD) Glasgow coma score & 12 & 10 & 2 \\
Abnormal EEG & 3 & 6 & 1 \\
Abnormal tomogram/MRI & & &
\end{tabular}

MRI, magnetic resonance imaging.
During the years 1984-92, 18 (64\%) patients were diagnosed based on CSF ELISA increased titres, and from 1992 to 1995,10 $(36 \%)$ additional patients were diagnosed based on PCR to detect HSV.

Of 28 children included in the series, two (7\%) died, and $26(93 \%)$ survived, of whom 16 $(57 \%)$ were left with no neurological sequelae and $10(36 \%)$ remained with persistent neurological sequelae.

Two patients aged 7 and 9 years died during the acute course of their disease. Postmortem examinations done in these cases showed strongly positive focal peroxidase staining with specific HSV antibodies in the cells, and typing with monoclonal antibodies confirmed the diagnosis of HSV type I encephalitis.

Table 2 compares the clinical characteristics on admission of patients with HSE in correlation to their long term outcome. There were no significant differences between the two groups of survivors, with and without neurological sequelae, and the patients who died, when the mean age, duration of symptoms, presence of seizures on admission, and the number of abnormal EEGs and neuroimaging studies were compared. Only the mean Glasgow coma score was significantly lower in the patients who died compared with the two survivor groups ( $p<0.001)$.

In two patients, recurrence of the disease was observed four and six days, respectively after treatment with aciclovir. A second course of aciclovir treatment was given at a dose of 30 $\mathrm{mg} / \mathrm{kg} /$ day for a further 10 days. Clinical improvement was seen after a few days, but the two patients were left with significant long term neurological sequelae. All the patients who survived were followed up in the paediatric neurology clinic. The follow up period ranged between 2-12 years with a mean follow up of 5.5 years.

In 10 patients neurological sequelae were observed, including cognitive dysfunction with IQ scores less than $70(n=4)$, personality changes $(n=4)$, speech abnormalities $(n=2)$, motor skill disturbances $(n=5)$, and epileptic seizures $(n=4)$. All these sequelae were observed during the first year of follow up, except for two patients who presented with epileptic disorders four and five years, respectively after the HSE.

\section{Discussion}

For more than a decade, aciclovir has been accepted as the drug of choice for treating HSE in adults and children. ${ }^{8}$ Two large prospective randomised studies comparing this drug with vidarabine proved its superiority to significantly improve both mortality and morbidity. ${ }^{6}{ }^{9}$

Children were included in these studies but in small numbers. Eight patients in one study, ${ }^{6}$ and six patients in another, ${ }^{9}$ were treated with aciclovir. To the best of our knowledge, our study describing 28 children with HSE treated with aciclovir is most probably the largest series of its kind published so far.

This study provides further confirmation that age and Glasgow coma score are the most 
Table 3 Morbidity and mortality in HSE

\begin{tabular}{lccllc}
\hline & $\begin{array}{l}\text { Skoldenberg } \\
\text { et al }\end{array}$ & $\begin{array}{l}\text { Whitley }(\%) \\
\text { et al } l^{\star}(\%)\end{array}$ & $\begin{array}{l}\text { Kimura et al } \\
(\%)\end{array}$ & $\begin{array}{l}\text { Cameron } \\
\text { et al }^{7}(\%)\end{array}$ & $\begin{array}{l}\text { Present study } \\
(\%)\end{array}$ \\
\hline Morbidity & $15(55.5)$ & $12(36.3)$ & $1(8.3)$ & - & $16(57)$ \\
$\quad$ Minor & $3(11.1)$ & $6(18.2)$ & $3(25)$ & - & $10(36)$ \\
$\quad \begin{array}{l}\text { Moderate } \\
\text { Severe }\end{array}$ & $4(14.8)$ & $9(27.3)$ & $7(58.3)$ & $6(100)$ & - \\
Mortality & $5(18.5)$ & $6(18.2)$ & $1(8.3)$ & - & $2(7)$ \\
Total & $27(100)$ & $33(100)$ & $12(100) \dagger$ & $6(100)$ & $28(100)$ \\
\hline
\end{tabular}

${ }^{\star}$ Most of the patients were adults. Only six $^{6}$ and eight children ${ }^{9}$ were included.

tAn additional three children were excluded because of short follow up period.

important variables influencing mortality and morbidity.

The overall mortality for aciclovir recipients in the above mentioned series were $19 \%$ and $28 \% .{ }^{69}$ The mortality rate for patients under 30 years in one series was $6 \%$ ( 1 of 18 ), whereas in our series the mortality rate was $7 \%$ (2 of 28). The Glasgow coma score influenced mortality and morbidity. Overall, a Glasgow coma score of less than 6 was observed in the two fatal cases, of $6-10$ in the patients with neurological sequelae, and over 10 in those patients with no neurological sequelae.

As has been emphasised by others, ${ }^{7}$ it is of the utmost importance to consider the diagnosis of HSE as early as possible and to start treatment even before obtaining the laboratory data that confirm or exclude the diagnosis. The clinical presentation of HSE is non-specific, especially in young children. Early symptoms may include malaise, mild irritability, or lethargy with no fever as the only manifestation. There are no characteristic findings in routine laboratory studies among children with HSE. CSF findings are abnormal in the majority of cases of HSE, typically with lymphocytosis and, at times, a high red cell count indicating a haemorrhagic process. However, lumbar puncture early in the illness may be normal, ${ }^{2}$ as we have shown in this study.

EEG findings are generally helpful in confirming the presence of encephalitis, ${ }^{27}$ specifically in younger children in whom history and examination findings are non-specific.

Neuroimaging studies may reveal characteristic low density contrast enhanced lesions in the temporal region, mass effect, oedema and haemorrhage. Unfortunately, early in the illness the tomogram is typically normal, ${ }^{7}$ as shown in $16(57 \%)$ of our patients. Magnetic resonance imaging studies are much more sensitive and usually are abnormal at the time of clinical presentation. ${ }^{10}$

Today, brain biopsy as a diagnostic procedure for HSE has been abandoned, and the PCR method is accepted as the preferred diagnostic test for HSE despite the risk of false positive $^{11}$ or false negative PCR results. ${ }^{12}$ Table 3 presents previous follow up studies in patients with HSE treated with aciclovir.

Data about morbidity in children with HSE are available from two studies. ${ }^{17}$ One described six children all left with significant disabilities, and in the second study, out of 15 children one died, seven were severely damaged, three moderately damaged, three were not evaluated, and only one child regained normal function.

Our results show a more favourable picture, with overall 16 out of 28 (64\%) children without any neurological sequelae after long term follow up.

Despite the major advantages in diagnosis and treatment of HSE, this disease is still associated with significant mortality and morbidity, emphasising the importance of considering HSE as a diagnostic possibility for children presenting with an encephalitic process and early treatment of suspected cases.

1 Kimura H, Aso K, Kuzushima K, et al. Relapse of herpes encephalitis in children. Pediatrics 1992;89:891-4.

2 Kohl S. Herpes simplex virus encephalitis in children. Pediatr Clin N Am 1988;35:465-83.

3 Kahlon J, Chetteriee S, Lakeman FD, et al. Detection of Kahlon J, Chetterjee S, Lakeman FD, et al. Detection of
antibodies to herpes simplex encephalitis. F Infect Dis 1987; 155:38-44.

4 Hanley DF, Johnson RT, Whitley RJ. Yes, brain biopsy should be prerequisite for herpes simplex encephalitis treatment. Arch Neurol 1987;44:1289-90.

5 Fishman RD. No, brain biopsy need not be done in every patient suspected of having herpes simplex encephalitis. Arch Neurol 1987;44:1291-2.

6 Whitley RJ, Alford CA, Hirch MS, et al. Vidarabine verus acyclovir therapy in herpes simplex encephalitis. $N$ Engl $\mathcal{F}$ Med 1986;314:144-9.

7 Cameron PD, Wallace SJ, Munro J. Herpes simplex virus encephalitis: problems in diagnosis. Develop Med Child Neurol 1992;34:134-40.

8 Whitley RJ, Ganann JW, Jr. Acyclovir: a decade later. N Engl f Med 1992;327:782-9.

9 Skoldenberg B, Alestig K, Burman L, et al. Acyclovir versus vidarabine in herpes simplex encephalitis. Lancet 1984;ii: 707-11.

10 Bale JF, Andersen RD, Grose C. Magnetic resonance imaging of the brain in childhood herpes virus infections. Pediatr Infect Dis f 1987;6:644-7.

11 Kwok S, Higuchi R. Avoiding false positives with PCR. Nature 1989;339:237-8.

12 Bowley AH, Whitley RJ, Lakerman FD, Wolinsky SM. Rapid detection of herpes-simplex-virus DNA in cerebrospinal fluid of patients with herpes simplex encephalitis. Lancet 1990;335:440-1. 